\title{
A CLASS OF ABSTRACT BOUNDARY VALUE PROBLEMS WITH LOCALLY DEFINITIZABLE FUNCTIONS IN THE BOUNDARY CONDITION
}

\author{
Jussi Behrndt
}

Preprint No. 2004/28 


\title{
A CLASS OF ABSTRACT BOUNDARY VALUE PROBLEMS WITH LOCALLY DEFINITIZABLE FUNCTIONS IN THE BOUNDARY CONDITION
}

\author{
Jussi Behrndt
}

\begin{abstract}
For a class of boundary value problems where the spectral parameter appears in the boundary condition in the form of a locally definitizable function linearizations are constructed and their local spectral properties are investigated.
\end{abstract}

\section{INTRODUCTION}

In this paper we study a class of boundary value problems with eigenvalue dependent boundary conditions. Let $A$ be a closed symmetric operator or relation with defect one in a separable Krein space $\mathcal{K}$, let $\left\{\mathbb{C}, \Gamma_{0}, \Gamma_{1}\right\}$ be a boundary value space for the adjoint $A^{+}$and let $\tau$ be a function locally holomorphic in some open subset of the extended complex plane which is symmetric with respect to the real line such that $\tau(\bar{\lambda})=\overline{\tau(\lambda)}$ holds. We investigate boundary value problems of the following form: For a given $k \in \mathcal{K}$ find a vector $\hat{f}=\left(\begin{array}{c}f \\ f^{\prime}\end{array}\right) \in A^{+}$such that

$$
f^{\prime}-\lambda f=k \quad \text { and } \quad \tau(\lambda) \Gamma_{0} \hat{f}+\Gamma_{1} \hat{f}=0
$$

holds. Under additional assumptions on $\tau$ and $A$ a solution of this problem can be obtained with the help of the compressed resolvent of a selfadjoint extension $\widetilde{A}$ of $A$ which acts in a larger Krein space. Making use of the coupling method from [6] we construct this so-called linearization and we study the spectral properties of $\widetilde{A}$ which are closely connected with the solvability of (1.1).

More precisely, let $\Omega$ be some domain in $\overline{\mathbb{C}}$ symmetric with respect to the real line such that $\Omega \cap \overline{\mathbb{R}} \neq \emptyset$ and the intersections of $\Omega$ with the upper and lower open half-planes are simply connected. We will assume that the selfadjoint extension $A_{0}:=\operatorname{ker} \Gamma_{0}$ of $A$ is definitizable over $\Omega$, i.e. for every subdomain $\Omega^{\prime}$ of $\Omega$ with the same properties as $\Omega, \overline{\Omega^{\prime}} \subset \Omega$, there exists a selfadjoint projection $E$ which reduces $A_{0}$ such that $A_{0} \cap(E \mathcal{K})^{2}$ is definitizable in the 
Krein space $E \mathcal{K}$ and $\Omega^{\prime}$ belongs to the resolvent set of $A_{0} \cap((1-E) \mathcal{K})^{2}$. With the help of approximative eigensequences or the local spectral function of $A_{0}$ the spectral points of $A_{0}$ in $\Omega \cap \overline{\mathbb{R}}$ can be classified in points of positive and negative type and critical points (cf. [13], [16]).

Further we assume that $\tau$ is a function which is definitizable in $\Omega$, that is for every domain $\Omega^{\prime}$ as $\Omega, \overline{\Omega^{\prime}} \subset \Omega$, the function $\tau$ can be written as the sum of a definitizable function (cf. [14], [15]) and a function holomorphic on $\Omega^{\prime}$. Similarly to selfadjoint operators and relations definitizable over $\Omega$ the points in $\Omega \cap \overline{\mathbb{R}}$ can be classified in points of positive and negative type and critical points. It was shown in [17] that $\tau$ can be represented with a selfadjoint relation $T_{0}$ definitizable over $\Omega^{\prime}$ in some Krein space $\mathcal{H}$ such that the sign types of $\tau$ and $T_{0}$ coincide in $\Omega^{\prime} \cap \overline{\mathbb{R}}$.

If, in addition, the sign types of $A_{0}$ and $\tau$ are "compatible" in $\Omega \cap \overline{\mathbb{R}}$ (see Definition 2.8) the selfadjoint relation $A_{0} \times T_{0}$ in the Krein space $\mathcal{K} \times \mathcal{H}$ is definitizable over $\Omega^{\prime}$. The linearization $\widetilde{A}$ of the boundary value problem (1.1) turns out to be a two dimensional perturbation in resolvent sense of $A_{0} \times T_{0}$. Under some additional minimality assumptions on the selfadjoint relations $A_{0}$ and $T_{0}$ and with the help of a recent result of T.Ya. Azizov and P. Jonas which states that the inverse of a matrix-valued locally definitizable function is again locally definitizable we prove in Theorem 3.6 that $\widetilde{A}$ is definitizable over $\Omega^{\prime}$.

The paper is organized as follows. In Section 2 we introduce the necessary notations and we recall the definitions of locally definitizable operators and relations and locally definitizable functions which can be found in e.g. [13], [16] and [17]. Section 3 deals with boundary value problems of the form (1.1). After some preparatory work in Sections 3.1 and 3.2 we formulate and prove the main result in Section 3.3: The linearization $\widetilde{A}$ of the eigenvalue dependent boundary value problem (1.1) is locally definitizable.

I thank P. Jonas for encouragement and critical help in the preparation of the manuscript.

\section{Locally Definitizable Selfadjoint Relations and Locally Definitizable Functions}

\subsection{Notations And Definitions}

Let $(\mathcal{K},[\cdot, \cdot])$ be a separable Krein space. We study linear relations in $\mathcal{K}$, that is, linear subspaces of $\mathcal{K}^{2}$. The set of all closed linear relations in $\mathcal{K}$ is denoted by $\widetilde{\mathcal{C}}(\mathcal{K})$. Linear operators in $\mathcal{K}$ are viewed as linear relations via their graphs. For the usual definitions of the linear operations with relations, the 
inverse etc., we refer to [9]. We denote the sum (direct sum) of subspaces in $\mathcal{K}^{2}$ by $\boldsymbol{+}$ (resp. $\left.\dot{\boldsymbol{+}}\right)$. The linear space of bounded linear operators defined on a Krein space $\mathcal{K}_{1}$ with values in a Krein space $\mathcal{K}_{2}$ is denoted by $\mathcal{L}\left(\mathcal{K}_{1}, \mathcal{K}_{2}\right)$. In the case $\mathcal{K}:=\mathcal{K}_{1}=\mathcal{K}_{2}$ we simply write $\mathcal{L}(\mathcal{K})$.

If $\left(\mathcal{H},[\cdot, \cdot]_{\mathcal{H}}\right)$ is another separable Krein space the elements of $\mathcal{K} \times \mathcal{H}$ will be written in the form $\{k, h\}, k \in \mathcal{K}, h \in \mathcal{H} . \mathcal{K} \times \mathcal{H}$ equipped with the inner product $[\cdot, \cdot]_{\mathcal{K} \times \mathcal{H}}$ defined by

$$
\left[\{k, h\},\left\{k^{\prime}, h^{\prime}\right\}\right]_{\mathcal{K} \times \mathcal{H}}:=\left[k, k^{\prime}\right]+\left[h, h^{\prime}\right]_{\mathcal{H}}, \quad k, k^{\prime} \in \mathcal{K}, \quad h, h^{\prime} \in \mathcal{H},
$$

is also a Krein space. If $S$ is a relation in $\mathcal{K}$ and $T$ is a relation in $\mathcal{H}$ we shall write $S \times T$ for the direct product of $S$ and $T$ which is a relation in $\mathcal{K} \times \mathcal{H}$,

$$
S \times T=\left\{\left(\begin{array}{c}
\{s, t\} \\
\left\{s^{\prime}, t^{\prime}\right\}
\end{array}\right) \mid\left(\begin{array}{c}
s \\
s^{\prime}
\end{array}\right) \in S,\left(\begin{array}{c}
t \\
t^{\prime}
\end{array}\right) \in T\right\} .
$$

For the pair $\left(\begin{array}{c}\{s, t\} \\ \left\{s^{\prime}, t^{\prime}\right\}\end{array}\right)$ on the right hand side of $(2.1)$ we shall also write $\{\hat{s}, \hat{t}\}$, where $\hat{s}=\left(\begin{array}{c}s \\ s^{\prime}\end{array}\right), \hat{t}=\left(\begin{array}{c}t \\ t^{\prime}\end{array}\right)$.

Let $S$ be a closed linear relation in $\mathcal{K}$. The resolvent set $\rho(S)$ of $S$ is the set of all $\lambda \in \mathbb{C}$ such that $(S-\lambda)^{-1} \in \mathcal{L}(\mathcal{K})$, the spectrum $\sigma(S)$ of $S$ is the complement of $\rho(S)$ in $\mathbb{C}$. The extended spectrum $\widetilde{\sigma}(S)$ of $S$ is defined by $\widetilde{\sigma}(S)=\sigma(S)$ if $S \in \mathcal{L}(\mathcal{K})$ and $\widetilde{\sigma}(S)=\sigma(S) \cup\{\infty\}$ otherwise. We say that $\lambda \in \mathbb{C}$ belongs to the approximate point spectrum of $S$, denoted by $\sigma_{a p}(S)$, if there exists a sequence $\left(\begin{array}{l}x_{n} \\ y_{n}\end{array}\right) \in S, n=1,2, \ldots$, such that $\left\|x_{n}\right\|=1$ and $\lim _{n \rightarrow \infty}\left\|y_{n}-\lambda x_{n}\right\|=0$. The extended approximate point spectrum $\widetilde{\sigma}_{a p}(S)$ of $S$ is defined by

$$
\tilde{\sigma}_{a p}(S):=\left\{\begin{array}{lll}
\sigma_{a p}(S) \cup\{\infty\} & \text { if } & 0 \in \sigma_{a p}\left(S^{-1}\right) \\
\sigma_{a p}(S) & \text { if } & 0 \notin \sigma_{a p}\left(S^{-1}\right)
\end{array} .\right.
$$

We remark, that the boundary points of $\widetilde{\sigma}(S)$ in $\overline{\mathbb{C}}$ belong to $\widetilde{\sigma}_{a p}(S)$.

Next we recall the definitions of the spectra of positive and negative type of a closed linear relation (see [16]). For equivalent descriptions of the spectra of positive and negative type we refer to [16, Theorem 3.18].

Definition 2.1. Let $S$ be a closed linear relation in $\mathcal{K}$. A point $\lambda \in \sigma_{a p}(S)$ is said to be of positive type (negative type) with respect to $S$, if for every sequence $\left(\begin{array}{l}x_{n} \\ y_{n}\end{array}\right) \in S, n=1,2 \ldots$, with $\left\|x_{n}\right\|=1, \lim _{n \rightarrow \infty}\left\|y_{n}-\lambda x_{n}\right\|=0$ we have

$$
\liminf _{n \rightarrow \infty}\left[x_{n}, x_{n}\right]>0 \quad\left(\text { resp. } \limsup _{n \rightarrow \infty}\left[x_{n}, x_{n}\right]<0\right) .
$$

If $\infty \in \widetilde{\sigma}_{a p}(S), \infty$ is said to be of positive type (negative type) with respect to $S$ if 0 is of positive (resp. negative) type with respect to $S^{-1}$.

An open subset $\Delta$ of $\overline{\mathbb{R}}$ is said to be of positive type (negative type) with respect to $S$ if each point $\lambda \in \Delta \cap \widetilde{\sigma}(S)$ is of positive (resp. negative) type with 
respect to $S . \Delta$ is called of definite type with respect to $S$ if $\Delta$ is of positive or negative type with respect to $S$.

Let $A$ be a linear relation in $\mathcal{K}$. The adjoint relation $A^{+} \in \widetilde{\mathcal{C}}(\mathcal{K})$ is defined as

$$
A^{+}:=\left\{\left(\begin{array}{c}
h \\
h^{\prime}
\end{array}\right) \mid\left[g^{\prime}, h\right]=\left[g, h^{\prime}\right] \text { for all } \quad\left(\begin{array}{c}
g \\
g^{\prime}
\end{array}\right) \in A\right\} .
$$

$A$ is said to be symmetric (selfadjoint) if $A \subset A^{+}$(resp. $A=A^{+}$).

For a selfadjoint relation $A$ in $\mathcal{K}$ the points of definite type introduced in Definition 2.1 are real. In fact, if e.g. $\lambda \neq \infty$ is of positive type with respect to $A$, and $\left(\begin{array}{l}x_{n} \\ y_{n}\end{array}\right) \in A$ is a sequence with $\left\|x_{n}\right\|=1$ and $\lim _{n \rightarrow \infty}\left\|y_{n}-\lambda x_{n}\right\|=0$, then

$$
|\operatorname{Im} \lambda| \liminf _{n \rightarrow \infty}\left[x_{n}, x_{n}\right]=\liminf _{n \rightarrow \infty}\left|\operatorname{Im}\left[y_{n}-\lambda x_{n}, x_{n}\right]\right| \leq \lim _{n \rightarrow \infty}\left\|y_{n}-\lambda x_{n}\right\|=0
$$

implies $\lambda \in \mathbb{R}$.

\subsection{Locally Definitizable Selfadjoint Relations in Krein Spaces}

Let $\Omega$ be a domain in $\overline{\mathbb{C}}$ symmetric with respect to the real axis such that $\Omega \cap \overline{\mathbb{R}} \neq \emptyset$ and the intersections of $\Omega$ with the upper and lower open half-planes are simply connected.

Let $A_{0}$ be a selfadjoint relation in the Krein space $\mathcal{K}$ such that $\sigma\left(A_{0}\right) \cap$ $(\Omega \backslash \overline{\mathbb{R}})$ consists of isolated points which are poles of the resolvent of $A_{0}$, and no point of $\Omega \cap \overline{\mathbb{R}}$ is an accumulation point of the non-real spectrum of $A_{0}$. We say that $A_{0}$ belongs to the class $S^{\infty}(\Omega \cap \overline{\mathbb{R}})$, if for every open subset $\Delta$ in $\overline{\mathbb{R}}, \bar{\Delta} \subset \Omega \cap \overline{\mathbb{R}}$, there exists $m \geq 1, M>0$ and an open neighbourhood $\mathcal{U}$ of $\bar{\Delta}$ in $\overline{\mathbb{C}}$ such that

$$
\left\|\left(A_{0}-\lambda\right)^{-1}\right\| \leq M(1+|\lambda|)^{2 m-2}|\operatorname{Im} \lambda|^{-m}
$$

holds for all $\lambda \in \mathcal{U} \backslash \overline{\mathbb{R}}$.

Definition 2.2. Let $A_{0}$ be a selfadjoint relation in $\mathcal{K}$ and let $\Omega$ be a domain as above. $A_{0}$ is said to be definitizable over $\Omega$, if $A_{0} \in S^{\infty}(\Omega \cap \overline{\mathbb{R}})$ and every point $\mu \in \Omega \cap \overline{\mathbb{R}}$ has an open connected neighbourhood $I_{\mu}$ in $\overline{\mathbb{R}}$ such that both components of $I_{\mu} \backslash\{\mu\}$ are of definite type with respect to $A_{0}$.

The next theorem is a variant of [16, Theorem 4.8]. The simple modification of the proof is left to reader.

Theorem 2.3. Let $A_{0}$ be a selfadjoint relation in $\mathcal{K}$ and let $\Omega$ be a domain as above. $A_{0}$ is definitizable over $\Omega$ if and only if for every domain $\Omega^{\prime}$ with the same properties as $\Omega, \overline{\Omega^{\prime}} \subset \Omega$, there exists a selfadjoint projection $E$ in $\mathcal{K}$ such that $A_{0}$ can be decomposed in

$$
A_{0}=\left(A_{0} \cap(E \mathcal{K})^{2}\right) \dot{+}\left(A_{0} \cap((1-E) \mathcal{K})^{2}\right)
$$


and the following holds.

(i) $A_{0} \cap(E \mathcal{K})^{2}$ is a definitizable relation in the Krein space $E \mathcal{K}$.

(ii) $\tilde{\sigma}\left(A_{0} \cap((1-E) \mathcal{K})^{2}\right) \cap \Omega^{\prime}=\emptyset$.

Let $A_{0}$ be a selfadjoint relation in $\mathcal{K}$ definitizable over $\Omega$, let $\Omega^{\prime}$ be a domain with the same properties as $\Omega, \overline{\Omega^{\prime}} \subset \Omega$, and let $E$ be a selfadjoint projection with the properties as in Theorem 2.3. If $E^{\prime}$ is the spectral function of the definitizable selfadjoint relation $A_{0} \cap(E \mathcal{K})^{2}$ in the Krein space $E \mathcal{K}$ (cf. $[15$, page 71$],[10]$ and [18]), then the mapping

$$
\delta \mapsto E^{\prime}(\delta) E=: E_{A_{0}}(\delta)
$$

defined for all finite unions $\delta$ of connected subsets of $\Omega^{\prime} \cap \overline{\mathbb{R}}$ the endpoints of which belong to $\Omega^{\prime} \cap \overline{\mathbb{R}}$ and are of definite type with respect to $A_{0} \cap(E \mathcal{K})^{2}$, is the spectral function of $A_{0}$ on $\Omega^{\prime} \cap \overline{\mathbb{R}}$ (see [16, Section 3.4 and Remark 4.9]).

\subsection{Locally Definitizable Functions}

Let $\Omega$ be a domain as in the beginning of Section 2.2 and let $\tau$ be an $\mathcal{L}\left(\mathbb{C}^{n}\right)$-valued piecewise meromorphic function in $\Omega \backslash \overline{\mathbb{R}}$ which is symmetric with respect to the real line, that is $\tau(\bar{\lambda})=\tau(\lambda)^{*}$ for all points $\lambda$ of holomorphy of $\tau$. If, in addition, no point of $\Omega \cap \overline{\mathbb{R}}$ is an accumulation point of nonreal poles of $\tau$ we write $\tau \in M^{n \times n}(\Omega)$. The set of the points of holomorphy of $\tau$ in $\Omega \backslash \overline{\mathbb{R}}$ and all points $\mu \in \Omega \cap \mathbb{R}$ such that $\tau$ can be analytically continued to $\mu$ and the continuations from $\Omega \cap \mathbb{C}^{+}$and $\Omega \cap \mathbb{C}^{-}$coincide, is denoted by $\mathfrak{h}(\tau)$.

In the next definition we introduce the sign type of open subsets in $\Omega \cap \overline{\mathbb{R}}$ with respect to functions from the class $M^{n \times n}(\Omega)$ (see [17]).

Definition 2.4. Let $\tau \in M^{n \times n}(\Omega)$. An open subset $\Delta \subset \Omega \cap \overline{\mathbb{R}}$ is said to be of positive type with respect to $\tau$ if for every $x \in \mathbb{C}^{n}$ and every sequence $\left(\mu_{k}\right)$ of points in $\Omega \cap \mathbb{C}^{+} \cap \mathfrak{h}(\tau)$ which converges in $\overline{\mathbb{C}}$ to a point of $\Delta$ we have

$$
\liminf _{k \rightarrow \infty} \operatorname{Im}\left(\tau\left(\mu_{k}\right) x, x\right) \geq 0 .
$$

An open subset $\Delta \subset \Omega \cap \overline{\mathbb{R}}$ is said to be of negative type with respect to $\tau$ if $\Delta$ is of positive type with respect to $-\tau$. $\Delta$ is said to be of definite type with respect to $\tau$ if $\Delta$ is of positive or of negative type with respect to $\tau$.

Definition 2.5. A function $\tau \in M^{n \times n}(\Omega)$ is called definitizable in $\Omega$ if the following holds.

(i) Every point $\mu \in \Omega \cap \overline{\mathbb{R}}$ has an open connected neighbourhood $I_{\mu}$ in $\overline{\mathbb{R}}$ such that both components of $I_{\mu} \backslash\{\mu\}$ are of definite type with respect to $\tau$. 
(ii) For every open subset $\Delta$ in $\overline{\mathbb{R}}, \bar{\Delta} \subset \Omega \cap \overline{\mathbb{R}}$, there exists $m \geq 1, M>0$ and an open neighbourhood $\mathcal{U}$ of $\bar{\Delta}$ in $\overline{\mathbb{C}}$ such that

$$
\|\tau(\lambda)\| \leq M(1+|\lambda|)^{2 m}|\operatorname{Im} \lambda|^{-m}
$$

holds for all $\lambda \in \mathcal{U} \backslash \overline{\mathbb{R}}$.

In [17] it is shown that a function $\tau \in M^{n \times n}(\Omega)$ is definitizable in $\Omega$ if and only if for every finite union $\Delta$ of open connected subsets of $\overline{\mathbb{R}}$ such that $\bar{\Delta} \subset \Omega \cap \overline{\mathbb{R}}, \tau$ can be written as the sum of an $\mathcal{L}\left(\mathbb{C}^{n}\right)$-valued definitizable function and an $\mathcal{L}\left(\mathbb{C}^{n}\right)$-valued function which is locally holomorphic on $\bar{\Delta}$.

The following theorem will be used in Sections 3.2 and 3.3. It states that a locally definitizable function can be represented with a locally definitizable selfadjoint relation. A proof can be found in [17].

Theorem 2.6. Let $\tau$ be an $\mathcal{L}\left(\mathbb{C}^{n}\right)$-valued function definitizable in $\Omega$ and let $\Omega^{\prime}$ be a domain with the same properties as $\Omega, \overline{\Omega^{\prime}} \subset \Omega$. Then there exists a Krein space $\mathcal{H}$, a selfadjoint relation $T_{0}$ in $\mathcal{H}$ definitizable over $\Omega^{\prime}$ and a mapping $\gamma^{\prime} \in \mathcal{L}\left(\mathbb{C}^{n}, \mathcal{H}\right)$ with the following properties.

(a) $\rho\left(T_{0}\right) \cap \Omega^{\prime}=\mathfrak{h}(\tau) \cap \Omega^{\prime}$.

(b) For a fixed $\lambda_{0} \in \rho\left(T_{0}\right) \cap \Omega^{\prime}$ and all $\lambda \in \rho\left(T_{0}\right) \cap \Omega^{\prime}$

$$
\tau(\lambda)=\operatorname{Re} \tau\left(\lambda_{0}\right)+\gamma^{\prime+}\left(\left(\lambda-\operatorname{Re} \lambda_{0}\right)+\left(\lambda-\lambda_{0}\right)\left(\lambda-\bar{\lambda}_{0}\right)\left(T_{0}-\lambda\right)^{-1}\right) \gamma^{\prime}
$$

holds.

(c) For any finite union $\Delta$ of open connected subsets of $\overline{\mathbb{R}}, \bar{\Delta} \subset \Omega^{\prime} \cap \overline{\mathbb{R}}$, such that the boundary points of $\Delta$ are of definite type with respect to $\tau$ the spectral projection $E_{T_{0}}(\Delta)$ is defined. If $\Omega^{\prime \prime}$ is a domain with the same properties as $\Omega$ and $\Omega^{\prime}, \overline{\Omega^{\prime \prime}} \subset \Omega^{\prime}$, and if we set $E:=E_{T_{0}}(\Delta)+E_{T_{0}}\left(\overline{\Omega^{\prime \prime}} \backslash \overline{\mathbb{R}}\right)$, then the minimality condition

$$
E \mathcal{H}=\operatorname{clsp}\left\{\left(1+\left(\lambda-\lambda_{0}\right)\left(T_{0}-\lambda\right)^{-1}\right) E \gamma^{\prime} x \mid \lambda \in \rho\left(T_{0}\right) \cap \Omega^{\prime}, x \in \mathbb{C}^{n}\right\}
$$

is fulfilled. $\Delta$ is of positive (negative) type with respect to $\tau$ if and only if $\Delta$ is of positive (resp. negative) type with respect to $T_{0}$.

If $\tau$ and $T_{0}$ are as in Theorem 2.6 we shall say that $T_{0}$ is an $\Omega^{\prime}$-minimal representing relation for $\tau$.

Remark 2.7. Let $\tau$ be an $\mathcal{L}\left(\mathbb{C}^{n}\right)$-valued function definitizable in $\Omega$ and let $\Omega^{\prime}$ be a domain with the same properties as $\Omega, \overline{\Omega^{\prime}} \subset \Omega$. If, in addition, $\tau$ is the restriction of a definitizable function (see [14], [15]) or if, in addition, the boundary of $\Omega^{\prime}$ is contained in $\mathfrak{h}(\tau)$, then the selfadjoint relation $T_{0}$ in Theorem 2.6 can be chosen such that the minimality condition

$$
\mathcal{H}=\operatorname{clsp}\left\{\left(1+\left(\lambda-\lambda_{0}\right)\left(T_{0}-\lambda\right)^{-1}\right) \gamma^{\prime} x \mid \lambda \in \rho\left(T_{0}\right) \cap \Omega^{\prime}, x \in \mathbb{C}^{n}\right\}
$$


holds.

The next definition connects sign types of locally definitizable functions and sign types of spectral points of locally definitizable relations.

Definition 2.8. Let $\tau$ be an $\mathcal{L}\left(\mathbb{C}^{n}\right)$-valued function definitizable in $\Omega$ and let $A_{0}$ be a selfadjoint relation in the Krein space $\mathcal{K}$ which is definitizable over $\Omega$. We say that the sign types of $\tau$ and $A_{0}$ are $d$-compatible in $\Omega$ if for every point $\mu \in \Omega \cap \overline{\mathbb{R}}$ there exists an open connected neighbourhood $I_{\mu} \subset \Omega \cap \overline{\mathbb{R}}$ of $\mu$ such that each component of $I_{\mu} \backslash\{\mu\}$ is of the same sign type with respect to $\tau$ and $A_{0}$.

If $\tau$ is a function which is definitizable in $\Omega, \Omega^{\prime}$ is a domain as $\Omega, \overline{\Omega^{\prime}} \subset \Omega$, and $T_{0}$ is an $\Omega^{\prime}$-minimal representing relation for $\tau$ (see Theorem 2.6), then the sign types of $\tau$ and $T_{0}$ are $d$-compatible in $\Omega^{\prime}$.

\section{Boundary Value Problems with Locally Definitizable Functions in the Boundary Condition}

\subsection{Boundary Value Spaces and Weyl Functions Associated with Symmetric Relations in Krein Spaces}

Let $(\mathcal{K},[\cdot, \cdot])$ be a separable Krein space, let $J$ be a corresponding fundamental symmetry and let $A \in \widetilde{\mathcal{C}}(\mathcal{K})$ be a closed symmetric relation in $\mathcal{K}$. We say that $A$ has defect $m \in \mathbb{N} \cup\{\infty\}$, if both deficiency indices

$$
n_{ \pm}(J A)=\operatorname{dim} \operatorname{ker}\left((J A)^{*}-\bar{\lambda}\right), \quad \lambda \in \mathbb{C}^{ \pm},
$$

of the symmetric relation $J A$ in the Hilbert space $(\mathcal{K},[J \cdot, \cdot])$ are equal to $m$. We remark, that this is equivalent to the fact that there exists a selfadjoint extension of $A$ in $\mathcal{K}$ and that each selfadjoint extension $\hat{A}$ of $A$ in $\mathcal{K}$ satisfies $\operatorname{dim}(\hat{A} / A)=m$.

We shall use the so-called boundary value spaces for the description of the selfadjoint extensions of closed symmetric relations in Krein spaces. The following definition is taken from [5].

Definition 3.1. Let $A$ be a closed symmetric relation in the Krein space $(\mathcal{K},[\cdot, \cdot])$. We say that $\left\{\mathcal{G}, \Gamma_{0}, \Gamma_{1}\right\}$ is a boundary value space for $A^{+}$if $(\mathcal{G},(\cdot, \cdot))$ is a Hilbert space and there exist mappings $\Gamma_{0}, \Gamma_{1}: A^{+} \rightarrow \mathcal{G}$ such that $\Gamma=$ $\left(\begin{array}{l}\Gamma_{0} \\ \Gamma_{1}\end{array}\right): A^{+} \rightarrow \mathcal{G} \times \mathcal{G}$ is surjective, and the relation

$$
\left[f^{\prime}, g\right]-\left[f, g^{\prime}\right]=\left(\Gamma_{1} \hat{f}, \Gamma_{0} \hat{g}\right)-\left(\Gamma_{0} \hat{f}, \Gamma_{1} \hat{g}\right)
$$

holds for all $\hat{f}=\left(\begin{array}{c}f \\ f^{\prime}\end{array}\right), \hat{g}=\left(\begin{array}{c}g \\ g^{\prime}\end{array}\right) \in A^{+}$. 
In the following we recall some basic facts on boundary value spaces which can be found in e.g. [4] and [5]. For the Hilbert space case we refer to [11], [7] and [8]. Let $A$ be a closed symmetric relation in $\mathcal{K}$, let

$$
\mathcal{N}_{\lambda, A^{+}}:=\operatorname{ker}\left(A^{+}-\lambda\right)=\operatorname{ran}(A-\bar{\lambda})^{[\perp]}
$$

be the defect subspace of $A$ and let

$$
\hat{\mathcal{N}}_{\lambda, A^{+}}=\left\{\left(\begin{array}{c}
f_{\lambda} \\
\lambda f_{\lambda}
\end{array}\right) \mid f_{\lambda} \in \mathcal{N}_{\lambda, A^{+}}\right\} .
$$

When no confusion can arise we write $\mathcal{N}_{\lambda}$ and $\hat{\mathcal{N}}_{\lambda}$ instead of $\mathcal{N}_{\lambda, A^{+}}$and $\hat{\mathcal{N}}_{\lambda, A^{+}}$. If there exists a selfadjoint extension $A^{\prime}$ of $A$ such that $\rho\left(A^{\prime}\right) \neq \emptyset$ then we have

$$
A^{+}=A^{\prime} \dot{+} \hat{\mathcal{N}}_{\lambda} \quad \text { for all } \quad \lambda \in \rho\left(A^{\prime}\right)
$$

In this case there exists a boundary value space $\left\{\mathcal{G}, \Gamma_{0}, \Gamma_{1}\right\}$ for $A^{+}$such that ker $\Gamma_{0}=A^{\prime}$ (cf. [5]).

Let in the following $A,\left\{\mathcal{G}, \Gamma_{0}, \Gamma_{1}\right\}$ and $\Gamma$ be as in Definition 3.1. It follows that the mappings $\Gamma_{0}$ and $\Gamma_{1}$ are continuous. The selfadjoint extensions

$$
A_{0}:=\operatorname{ker} \Gamma_{0} \quad \text { and } \quad A_{1}:=\operatorname{ker} \Gamma_{1}
$$

of $A$ are transversal, i.e. $A_{0} \cap A_{1}=A$ and $A_{0}+A_{1}=A^{+}$. The mapping $\Gamma$ induces, via

$$
A_{\Theta}:=\Gamma^{-1} \Theta=\left\{\hat{f} \in A^{+} \mid \Gamma \hat{f} \in \Theta\right\}, \quad \Theta \in \widetilde{\mathcal{C}}(\mathcal{G}),
$$

a bijective correspondence $\Theta \mapsto A_{\Theta}$ between the set of all closed linear relations $\widetilde{\mathcal{C}}(\mathcal{G})$ in $\mathcal{G}$ and the set of closed extensions $A_{\Theta} \subset A^{+}$of $A$. In particular (3.1) gives a one-to-one correspondence between the symmetric (selfadjoint) extensions of $A$ and the symmetric (resp. selfadjoint) relations in $\mathcal{G}$. If $\Theta$ is a closed operator in $\mathcal{G}$, then the corresponding extension $A_{\Theta}$ of $A$ is determined by

$$
A_{\Theta}=\operatorname{ker}\left(\Gamma_{1}-\Theta \Gamma_{0}\right) .
$$

Assume that $\rho\left(A_{0}\right) \neq \emptyset$ and denote by $\pi_{1}$ the orthogonal projection onto the first component of $\mathcal{K}^{2}$. For every $\lambda \in \rho\left(A_{0}\right)$ we define the operators

$$
\gamma(\lambda)=\pi_{1}\left(\Gamma_{0} \mid \hat{\mathcal{N}}_{\lambda}\right)^{-1} \in \mathcal{L}(\mathcal{G}, \mathcal{K}) \text { and } M(\lambda)=\Gamma_{1}\left(\Gamma_{0} \mid \hat{\mathcal{N}}_{\lambda}\right)^{-1} \in \mathcal{L}(\mathcal{G}) .
$$

The functions $\lambda \mapsto \gamma(\lambda)$ and $\lambda \mapsto M(\lambda)$ are called the $\gamma$-field and Weyl function corresponding to $A$ and $\left\{\mathcal{G}, \Gamma_{0}, \Gamma_{1}\right\} . \gamma$ and $M$ are holomorphic on $\rho\left(A_{0}\right)$ and the relations

$$
\gamma(\zeta)=\left(1+(\zeta-\lambda)\left(A_{0}-\zeta\right)^{-1}\right) \gamma(\lambda)
$$

and

$$
M(\lambda)-M(\zeta)^{*}=(\lambda-\bar{\zeta}) \gamma(\zeta)^{+} \gamma(\lambda)
$$

hold for all $\lambda, \zeta \in \rho\left(A_{0}\right)$ (cf. [5]). 
If $\Theta \in \widetilde{\mathcal{C}}(\mathcal{G})$ and $A_{\Theta}$ is the corresponding extension of $A$ (see (3.1)), then a point $\lambda \in \rho\left(A_{0}\right)$ belongs to $\rho\left(A_{\Theta}\right)$ if and only if 0 belongs to $\rho(\Theta-M(\lambda))$. For $\lambda \in \rho\left(A_{\Theta}\right) \cap \rho\left(A_{0}\right)$ the well-known resolvent formula

$$
\left(A_{\Theta}-\lambda\right)^{-1}=\left(A_{0}-\lambda\right)^{-1}+\gamma(\lambda)(\Theta-M(\lambda))^{-1} \gamma(\bar{\lambda})^{+}
$$

holds (for a proof see e.g. [5]).

\subsection{Locally Definitizable Functions as Weyl Functions of Symmetric Relations}

Let, as in Section $2.2, \Omega$ be a domain in $\overline{\mathbb{C}}$ symmetric with respect to the real axis such that $\Omega \cap \overline{\mathbb{R}} \neq \emptyset$ and the intersections of $\Omega$ with the upper and lower open half-planes are simply connected.

In the next proposition we consider boundary value spaces and Weyl functions associated with symmetric relations in Krein spaces which have the additional property that there exists a locally definitizable selfadjoint extension. We restrict ourselves to the case of defect one.

Proposition 3.2. Let $A$ be a closed symmetric relation with defect one in the Krein space $\mathcal{K}$ and assume that there exists a selfadjoint extension $A_{0}$ of $A$ which is definitizable over $\Omega$. Let $\left\{\mathbb{C}, \Gamma_{0}, \Gamma_{1}\right\}$ be a boundary value space for $A^{+}$such that $A_{0}=\operatorname{ker} \Gamma_{0}$. Then the corresponding Weyl function $M$ is definitizable in $\Omega$. If $\Delta$ is an open subset of $\Omega \cap \overline{\mathbb{R}}$ which is of positive (negative) type with respect to $A_{0}$, then $\Delta$ is of positive (resp. negative) type with respect to $M$.

Proof. As $A_{0}$ is a selfadjoint relation definitizable over $\Omega$ it follows that the Weyl function $M$ corresponding to $A$ and $\left\{\mathbb{C}, \Gamma_{0}, \Gamma_{1}\right\}$ is piecewise meromorphic in $\Omega \backslash \overline{\mathbb{R}}$ and no point of $\Omega \cap \overline{\mathbb{R}}$ is an accumulation point of nonreal poles of $M$. Let $\lambda_{0} \in \rho\left(A_{0}\right)$. Making use of (3.3) and (3.4) we obtain that $M$ is symmetric with respect to the real axis, $\operatorname{Im} M\left(\lambda_{0}\right)=\operatorname{Im} \lambda_{0} \gamma\left(\lambda_{0}\right)^{+} \gamma\left(\lambda_{0}\right)$ and

$$
\begin{aligned}
& M(\lambda)=\overline{M\left(\lambda_{0}\right)}+\left(\lambda-\bar{\lambda}_{0}\right) \gamma\left(\lambda_{0}\right)^{+} \gamma(\lambda) \\
& =\operatorname{Re} M\left(\lambda_{0}\right)-i \operatorname{Im} \lambda_{0} \gamma\left(\lambda_{0}\right)^{+} \gamma\left(\lambda_{0}\right) \\
& \quad+\gamma\left(\lambda_{0}\right)^{+}\left(\lambda-\bar{\lambda}_{0}+\left(\lambda-\lambda_{0}\right)\left(\lambda-\bar{\lambda}_{0}\right)\left(A_{0}-\lambda\right)^{-1}\right) \gamma\left(\lambda_{0}\right) \\
& =\operatorname{Re} M\left(\lambda_{0}\right)+\gamma\left(\lambda_{0}\right)^{+}\left(\left(\lambda-\operatorname{Re} \lambda_{0}\right)+\left(\lambda-\lambda_{0}\right)\left(\lambda-\bar{\lambda}_{0}\right)\left(A_{0}-\lambda\right)^{-1}\right) \gamma\left(\lambda_{0}\right)
\end{aligned}
$$

holds for all $\lambda \in \rho\left(A_{0}\right)$. Since $A_{0}$ belongs to the class $S^{\infty}(\Omega \cap \overline{\mathbb{R}})$ it follows that $M$ fulfils the second condition in Definition 2.5. Let $\mu \in \Omega \cap \overline{\mathbb{R}}$ and let $I_{\mu} \subset \Omega \cap \overline{\mathbb{R}}$ be an open connected neighbourhood of $\mu$ in $\overline{\mathbb{R}}$ such that both components of $I_{\mu} \backslash\{\mu\}$ are of definite type with respect to $A_{0}$. By [16, Theorem 3.18] a component of $I_{\mu} \backslash\{\mu\}$ is of positive (negative) type with respect to $A_{0}$ 
if and only if it is of positive (resp. negative) type with respect to the function

$$
\lambda \mapsto\left(\lambda-\operatorname{Re} \lambda_{0}\right)+\left(\lambda-\lambda_{0}\right)\left(\lambda-\bar{\lambda}_{0}\right)\left(A_{0}-\lambda\right)^{-1} .
$$

Therefore both components of $I_{\mu} \backslash\{\mu\}$ are of the same sign type with respect to $A_{0}$ and $M$ and it follows that $M$ is definitizable in $\Omega$.

The next theorem is a variant of [3, Theorem 3.3]. For the convenience of the reader we sketch the proof.

Theorem 3.3. Let $\tau$ be a complex valued function definitizable in $\Omega$ and assume that $\tau$ is not identically equal to a constant. Let $\Omega^{\prime}$ be a domain with the same properties as $\Omega$ and $\overline{\Omega^{\prime}} \subset \Omega$. Then there exists a Krein space $\mathcal{H}$, a closed symmetric relation $T$ with defect one in $\mathcal{H}$ and a boundary value space $\left\{\mathbb{C}, \Gamma_{0}^{\prime}, \Gamma_{1}^{\prime}\right\}$ for $T^{+}$such that $\tau$ coincides with the corresponding Weyl function on $\Omega^{\prime}$ and $T_{0}:=\operatorname{ker} \Gamma_{0}^{\prime}$ is an $\Omega^{\prime}$-minimal representing relation for $\tau$.

Sketch of the proof of Theorem 3.3. Let $\tau$ be represented with an $\Omega^{\prime}$-minimal selfadjoint relation $T_{0}$ in a Krein space $\mathcal{H}$ as in Theorem 2.6. Let $\gamma^{\prime} \in \mathcal{L}(\mathbb{C}, \mathcal{H})$ be as in Theorem 2.6 and fix some $\lambda_{0} \in \Omega^{\prime} \cap \mathfrak{h}(\tau)$. For all $\lambda \in \Omega^{\prime} \cap \mathfrak{h}(\tau)$ we define

$$
\gamma^{\prime}(\lambda):=\left(1+\left(\lambda-\lambda_{0}\right)\left(T_{0}-\lambda\right)^{-1}\right) \gamma^{\prime} \in \mathcal{L}(\mathbb{C}, \mathcal{H})
$$

The linear functional $\gamma^{\prime}(\lambda) c \mapsto c$ defined on $\operatorname{ran} \gamma^{\prime}(\lambda)$ is denoted by $\gamma^{\prime}(\lambda)^{(-1)}$. The closed symmetric relation

$$
T:=\left\{\left(\begin{array}{l}
f \\
g
\end{array}\right) \in T_{0} \mid\left[g-\bar{\mu} f, \gamma^{\prime}(\mu) 1\right]=0\right\}
$$

has defect one and does not depend on the choice of $\mu \in \Omega^{\prime} \cap \mathfrak{h}(\tau)$. For every $\lambda \in \Omega^{\prime} \cap \mathfrak{h}(\tau)$ we write the elements $\hat{f} \in T^{+}$in the form $\hat{f}=\left(\begin{array}{c}f_{0} \\ f_{0}^{\prime}\end{array}\right)+\left(\begin{array}{c}f_{\lambda} \\ \lambda f_{\lambda}\end{array}\right)$, where $\left(\begin{array}{l}f_{0} \\ f_{0}^{\prime}\end{array}\right) \in T_{0}$ and $f_{\lambda} \in \operatorname{ran} \gamma^{\prime}(\lambda)=\mathcal{N}_{\lambda, T^{+}}$. As in the proof of [3, Theorem 3.3] (see also $\left[7\right.$, Theorem 1]) one verifies that $\left\{\mathbb{C}, \Gamma_{0}^{\prime}, \Gamma_{1}^{\prime}\right\}$, where

$$
\begin{aligned}
& \Gamma_{0}^{\prime} \hat{f}:=\gamma^{\prime}(\lambda)^{(-1)} f_{\lambda}, \\
& \Gamma_{1}^{\prime} \hat{f}:=\gamma^{\prime}(\lambda)^{+}\left(f_{0}^{\prime}-\bar{\lambda} f_{0}\right)+\tau(\lambda) \gamma^{\prime}(\lambda)^{(-1)} f_{\lambda},
\end{aligned}
$$

is a boundary value space for $T^{+}$and the corresponding Weyl function coincides with $\tau$ on $\Omega^{\prime}$.

Remark 3.4. Let the function $\tau$ be definitizable in $\Omega$ and assume that $\tau$ is not identically equal to a constant. Let $\Omega^{\prime}$ be a domain with the same properties as $\Omega, \overline{\Omega^{\prime}} \subset \Omega$, and assume that $\tau$ is the restriction of a definitizable function 
or that the boundary of $\Omega^{\prime}$ is contained in $\mathfrak{h}(\tau)$. If we choose $T_{0}$ as in Remark 2.7 and $T \subset T_{0} \subset T^{+}$as in Theorem 3.3, then the condition

$$
\mathcal{H}=\operatorname{clsp}\left\{\operatorname{ran} \gamma^{\prime}(\lambda) \mid \lambda \in \rho\left(T_{0}\right) \cap \Omega^{\prime}\right\}=\operatorname{clsp}\left\{\mathcal{N}_{\lambda, T^{+}} \mid \lambda \in \rho\left(T_{0}\right) \cap \Omega^{\prime}\right\}
$$

is fulfilled.

In the following proposition we use Definition 2.8. The statements will be useful in the proof of our main result in Section 3.3.

Proposition 3.5. Let $A$ be a closed symmetric relation with defect one in the Krein space $\mathcal{K}$, let $\left\{\mathbb{C}, \Gamma_{0}, \Gamma_{1}\right\}$ be a boundary value space for $A^{+}$and denote by $M$ the corresponding Weyl function. Assume that the selfadjoint relation $A_{0}=\operatorname{ker} \Gamma_{0}$ is definitizable over $\Omega$ and let $\tau$ be a function which is definitizable in $\Omega$ such that the sign types of $\tau$ and $A_{0}$ are $d$-compatible in $\Omega$.

Let $\Omega^{\prime}$ be a domain with the same properties as $\Omega, \overline{\Omega^{\prime}} \subset \Omega$, and let $T_{0}$ be an $\Omega^{\prime}$-minimal representing relation for $\tau$ in some Krein space $\mathcal{H}$ (see Theorem 2.6). Then the following holds.

(i) The selfadjoint relation $A_{0} \times T_{0} \in \widetilde{\mathcal{C}}(\mathcal{K} \times \mathcal{H})$ is definitizable over $\Omega^{\prime}$ and the sign types of $A_{0} \times T_{0}$ and the functions $\tau$ and $M$ are $d$-compatible in $\Omega^{\prime}$.

(ii) $M+\tau$ is definitizable in $\Omega$.

(iii) If $\tau(\eta) \neq 0$ and $(M+\tau)\left(\eta^{\prime}\right) \neq 0$ for some $\eta, \eta^{\prime} \in \Omega$, then the functions

$$
\lambda \mapsto\left(\begin{array}{cc}
M(\lambda) & 0 \\
0 & -\tau(\lambda)^{-1}
\end{array}\right) \quad \text { and } \quad \lambda \mapsto-\left(\begin{array}{cc}
M(\lambda) & -1 \\
-1 & -\tau(\lambda)^{-1}
\end{array}\right)^{-1}
$$

are definitizable in $\Omega$ and their sign types are $d$-compatible with the sign types of the selfadjoint relation $A_{0} \times T_{0}$ in $\Omega^{\prime}$.

Proof. (i) Since $A_{0}$ and $T_{0}$ belong to $S^{\infty}(\Omega \cap \overline{\mathbb{R}})$ and $S^{\infty}\left(\Omega^{\prime} \cap \overline{\mathbb{R}}\right)$, respectively, we conclude that $A_{0} \times T_{0}$ belongs to $S^{\infty}\left(\Omega^{\prime} \cap \overline{\mathbb{R}}\right)$. Let $\mu \in \Omega^{\prime} \cap \overline{\mathbb{R}}$ and let $I_{\mu} \subset \Omega^{\prime} \cap \overline{\mathbb{R}}$ be an open connected neighbourhood of $\mu$ in $\overline{\mathbb{R}}$ such that each component of $I_{\mu} \backslash\{\mu\}$ is of the same sign type with respect to $A_{0}$ and $\tau$. As $T_{0}$ is an $\Omega^{\prime}$-minimal representing relation for $\tau$ both components of $I_{\mu} \backslash\{\mu\}$ are of definite type with respect to $A_{0} \times T_{0}$ and it follows that $A_{0} \times T_{0}$ is definitizable over $\Omega^{\prime}$.

The assumption that the sign types of $\tau$ and $A_{0}$ are $d$-compatibel in $\Omega$ implies that the sign types of $\tau$ and $A_{0} \times T_{0}$ as well as the sign types of $M$ and $A_{0} \times T_{0}$ are $d$-compatible in $\Omega$.

(ii) For $\mu \in \Omega \cap \overline{\mathbb{R}}$ we choose an open connected neighbourhood $I_{\mu} \subset \Omega \cap \overline{\mathbb{R}}$ of $\mu$ such that both components of $I_{\mu} \backslash\{\mu\}$ are of the same sign type with respect to $A_{0}$ and $\tau$. By Proposition 3.2 the sign types of $M$ are the same as of $A_{0}$ and therefore both components of $I_{\mu} \backslash\{\mu\}$ are of the same sign type with respect 
to $M+\tau$. The growth properties of $M$ and $\tau$ imply that $M+\tau$ fulfils the second condition in Definition 2.5 and therefore $M+\tau$ is definitizable in $\Omega$.

(iii) By [1, Theorem 2.3] the function $-\tau^{-1}$ is definitizable in $\Omega$ and it follows from the proof of $[1$, Theorem 2.3] that each point $\mu \in \Omega \cap \overline{\mathbb{R}}$ has an open connected neighbourhood $I_{\mu} \subset \Omega \cap \overline{\mathbb{R}}$ such that both components of $I_{\mu} \backslash\{\mu\}$ are of the same sign type with respect to $-\tau^{-1}$ and $\tau$. Therefore the sign types of $-\tau^{-1}$ and $A_{0}$ are $d$-compatible in $\Omega$. Now it is easy to see that the first function in (3.7) is definitizable in $\Omega$ and its sign types are $d$-compatible with the sign types of $A_{0} \times T_{0}$ in $\Omega^{\prime}$.

As the function

$$
\lambda \mapsto\left(\begin{array}{cc}
M(\lambda) & -1 \\
-1 & -\tau(\lambda)^{-1}
\end{array}\right)
$$

is also definitizable in $\Omega$ another application of [1, Theorem 2.3] shows that the second function in (3.7) is definitizable in $\Omega$ and its sign types are $d$-compatible with the sign types of $A_{0} \times T_{0}$ in $\Omega^{\prime}$.

\subsection{The Main Result}

In this section we investigate the spectral properties of so-called linearizations of a class of abstract eigenvalue dependent boundary value problems with locally definitizable functions in the boundary condition. Similar problems with a local variant of generalized Nevanlinna functions in the boundary condition have been considered in [3]. The main feature in Theorem 3.6 below is that the linearization turns out to be locally definitizable.

Theorem 3.6. Let $\Omega$ be a domain as in the beginning of Section 3.2 and let $A$ be a closed symmetric relation with defect one in the Krein space $\mathcal{K}$ such that $\mathcal{K}=\operatorname{clsp}\left\{\mathcal{N}_{\lambda, A^{+}} \mid \lambda \in \Omega\right\}$ holds. Assume that there exists a selfadjoint extension $A_{0}$ of $A$ which is definitizable over $\Omega$. Let $\left\{\mathbb{C}, \Gamma_{0}, \Gamma_{1}\right\}$ be a boundary value space for $A^{+}, A_{0}=\operatorname{ker} \Gamma_{0}$, and denote by $\gamma$ and $M$ the corresponding $\gamma$-field and Weyl function, respectively.

Let $\tau$ be a nonconstant function which is definitizable in $\Omega$, let $\Omega^{\prime}$ be a domain as $\Omega, \overline{\Omega^{\prime}} \subset \Omega$, choose $\mathcal{H}, T \subset T_{0} \subset T^{+}$and $\left\{\mathbb{C}, \Gamma_{0}^{\prime}, \Gamma_{1}^{\prime}\right\}$ as in Theorem 3.3 and assume that the condition $\mathcal{H}=\operatorname{clsp}\left\{\mathcal{N}_{\lambda, T^{+}} \mid \lambda \in \Omega^{\prime}\right\}$ is fulfilled.

Let the sign types of $\tau$ and $A_{0}$ be $d$-compatible in $\Omega$, assume that the function $M+\tau$ is not identically equal to zero and define

$$
\mathfrak{h}_{0}:=\mathfrak{h}(M) \cap \mathfrak{h}(\tau) \cap \mathfrak{h}\left(\tau^{-1}\right) \cap \mathfrak{h}\left((M+\tau)^{-1}\right) .
$$

Then the relation

$$
\widetilde{A}=\left\{\left\{\hat{f}_{1}, \hat{f}_{2}\right\} \in A^{+} \times T^{+} \mid \Gamma_{1} \hat{f}_{1}-\Gamma_{1}^{\prime} \hat{f}_{2}=\Gamma_{0} \hat{f}_{1}+\Gamma_{0}^{\prime} \hat{f}_{2}=0\right\}
$$


is a selfadjoint extension of $A$ in $\mathcal{K} \times \mathcal{H}$ which is definitizable over $\Omega^{\prime}$ and the sign types of $\widetilde{A}$ are $d$-compatible with the sign types of $\tau$ and $M$ in $\Omega^{\prime}$. The set $\Omega^{\prime} \backslash\left(\overline{\mathbb{R}} \cup \mathfrak{h}_{0}\right)$ is finite. For every $k \in \mathcal{K}$ and every $\lambda \in \mathfrak{h}_{0}$ a unique solution of the eigenvalue dependent boundary value problem

$$
f_{1}^{\prime}-\lambda f_{1}=k, \quad \tau(\lambda) \Gamma_{0} \hat{f}_{1}+\Gamma_{1} \hat{f}_{1}=0, \quad \hat{f}_{1}=\left(\begin{array}{l}
f_{1} \\
f_{1}^{\prime}
\end{array}\right) \in A^{+},
$$

is given by

$$
\begin{aligned}
& f_{1}=P_{\mathcal{K}}(\widetilde{A}-\lambda)^{-1}\{k, 0\}=\left(A_{0}-\lambda\right)^{-1} k-\gamma(\lambda)(M(\lambda)+\tau(\lambda))^{-1} \gamma(\bar{\lambda})^{+} k, \\
& f_{1}^{\prime}=\lambda f_{1}+k .
\end{aligned}
$$

Proof. 1. In this step we show that (3.10) is a solution of the boundary value problem (3.9). We follow the lines of [3, Proof of Theorem 4.1] (see also [6, $\S 5.2])$.

As the functions $M, \tau$ and $M+\tau$ are definitizable in $\Omega$ (see Proposition 3.5) [1, Theorem 2.3] implies that $-\tau^{-1}$ and $-(M+\tau)^{-1}$ are also definitizable in $\Omega$. Let $\Omega^{\prime}$ and $\mathfrak{h}_{0}$ be as in the assumptions of the theorem. As the nonreal poles of the functions $M, \tau, \tau^{-1}$ and $(M+\tau)^{-1}$ in $\Omega$ do not accumulate to $\Omega \cap \overline{\mathbb{R}}$ we conclude that the set $\Omega^{\prime} \backslash\left(\overline{\mathbb{R}} \cup \mathfrak{h}_{0}\right)$ is finite.

Let $\mathcal{H}, T \subset T^{+}$and $\left\{\mathbb{C}, \Gamma_{0}^{\prime}, \Gamma_{1}^{\prime}\right\}$ be as in Theorem 3.3. Then $\tau$ is the corresponding Weyl function and the selfadjoint relation $T_{0}=\operatorname{ker} \Gamma_{0}^{\prime}$ is definitizable over $\Omega^{\prime}$. By $\gamma^{\prime}$ we denote the $\gamma$-field corresponding to $\left\{\mathbb{C}, \Gamma_{0}^{\prime}, \Gamma_{1}^{\prime}\right\}$ and we set $T_{1}:=\operatorname{ker} \Gamma_{1}^{\prime}$. As $\left\{\mathbb{C}, \Gamma_{1}^{\prime},-\Gamma_{0}^{\prime}\right\}$ is a boundary value space for $T^{+}$with corresponding $\gamma$-field and Weyl function

$$
\lambda \mapsto \gamma^{\prime}(\lambda) \tau(\lambda)^{-1} \quad \text { and } \quad \lambda \mapsto-\tau(\lambda)^{-1},
$$

respectively, it follows without difficulty that $\left\{\mathbb{C}^{2}, \widetilde{\Gamma}_{0}, \widetilde{\Gamma}_{1}\right\}$, where $\widetilde{\Gamma}_{0}$ and $\widetilde{\Gamma}_{1}$ are mappings from $A^{+} \times T^{+}$into $\mathbb{C}^{2}$ defined by

$$
\widetilde{\Gamma}_{0}\left\{\hat{f}_{1}, \hat{f}_{2}\right\}:=\left(\begin{array}{l}
\Gamma_{0} \hat{f}_{1} \\
\Gamma_{1}^{\prime} \hat{f}_{2}
\end{array}\right) \quad \text { and } \quad \widetilde{\Gamma}_{1}\left\{\hat{f}_{1}, \hat{f}_{2}\right\}:=\left(\begin{array}{c}
\Gamma_{1} \hat{f}_{1} \\
-\Gamma_{0}^{\prime} \hat{f}_{2}
\end{array}\right)
$$

$\left\{\hat{f}_{1}, \hat{f}_{2}\right\} \in A^{+} \times T^{+}$, is a boundary value space for $A^{+} \times T^{+}$with corresponding $\gamma$-field

$$
\lambda \mapsto \widetilde{\gamma}(\lambda)=\left(\begin{array}{cc}
\gamma(\lambda) & 0 \\
0 & \gamma^{\prime}(\lambda) \tau(\lambda)^{-1}
\end{array}\right), \quad \lambda \in \mathfrak{h}(M) \cap \mathfrak{h}(\tau) \cap \mathfrak{h}\left(\tau^{-1}\right),
$$

and corresponding Weyl function

$$
\lambda \mapsto \widetilde{M}(\lambda)=\left(\begin{array}{cc}
M(\lambda) & 0 \\
0 & -\tau(\lambda)^{-1}
\end{array}\right), \quad \lambda \in \mathfrak{h}(M) \cap \mathfrak{h}(\tau) \cap \mathfrak{h}\left(\tau^{-1}\right) .
$$


The selfadjoint relation $\widetilde{A}$ in $\mathcal{K} \times \mathcal{H}$ corresponding to $\Theta=\left(\begin{array}{ll}0 & 1 \\ 1 & 0\end{array}\right) \in \mathcal{L}\left(\mathbb{C}^{2}\right)$ via (3.1) is given by

$$
\begin{aligned}
\widetilde{A} & =\operatorname{ker}\left(\widetilde{\Gamma}_{1}-\Theta \widetilde{\Gamma}_{0}\right) \\
& =\left\{\left\{\hat{f}_{1}, \hat{f}_{2}\right\} \in A^{+} \times T^{+} \mid \Gamma_{1} \hat{f}_{1}-\Gamma_{1}^{\prime} \hat{f}_{2}=\Gamma_{0} \hat{f}_{1}+\Gamma_{0}^{\prime} \hat{f}_{2}=0\right\} .
\end{aligned}
$$

For $\lambda \in \mathfrak{h}_{0}$ the resolvent of $\widetilde{A}$ can be written as

$$
(\widetilde{A}-\lambda)^{-1}=\left(\begin{array}{cc}
\left(A_{0}-\lambda\right)^{-1} & 0 \\
0 & \left(T_{1}-\lambda\right)^{-1}
\end{array}\right)+\widetilde{\gamma}(\lambda)(\Theta-\widetilde{M}(\lambda))^{-1} \widetilde{\gamma}(\bar{\lambda})^{+}
$$

(see (3.5)). Calculating $(\Theta-\widetilde{M}(\lambda))^{-1}$ one verifies that the compressed resolvent of $\widetilde{A}$ onto $\mathcal{K}$ is given by

$$
P_{\mathcal{K}}(\widetilde{A}-\lambda)^{-1} \mid \mathcal{K}=\left(A_{0}-\lambda\right)^{-1}-\gamma(\lambda)(M(\lambda)+\tau(\lambda))^{-1} \gamma(\bar{\lambda})^{+}, \quad \lambda \in \mathfrak{h}_{0} .
$$

For $k \in \mathcal{K}$ we set $f_{1}:=P_{\mathcal{K}}(\widetilde{A}-\lambda)^{-1}\{k, 0\}$ and $f_{2}:=P_{\mathcal{H}}(\widetilde{A}-\lambda)^{-1}\{k, 0\}$. Then

$$
\left(\begin{array}{c}
\left\{f_{1}, f_{2}\right\} \\
\left\{\lambda f_{1}+k, \lambda f_{2}\right\}
\end{array}\right) \in \widetilde{A} \subset A^{+} \times T^{+}
$$

and $\hat{f}_{1}:=\left(\begin{array}{c}f_{1} \\ \lambda f_{1}+k\end{array}\right) \in A^{+}, \hat{f}_{2}:=\left(\begin{array}{c}f_{2} \\ \lambda f_{2}\end{array}\right) \in \hat{\mathcal{N}}_{\lambda, T^{+}}$. From (3.13) and since $\tau$ is the Weyl function corresponding to $\left\{\mathbb{C}, \Gamma_{0}^{\prime}, \Gamma_{1}^{\prime}\right\}$ we get

$$
\Gamma_{1} \hat{f}_{1}=\Gamma_{1}^{\prime} \hat{f}_{2}=\tau(\lambda) \Gamma_{0}^{\prime} \hat{f}_{2}=-\tau(\lambda) \Gamma_{0} \hat{f}_{1}, \quad \lambda \in \mathfrak{h}_{0},
$$

and it follows that $\hat{f}_{1} \in A^{+}$is a solution of (3.9).

Let us verify that this solution $\hat{f}_{1} \in A^{+}$is unique. Assume that the vector $\hat{g}_{1}=\left(\begin{array}{c}g_{1} \\ \lambda g_{1}+k\end{array}\right) \in A^{+}$is also a solution of $(3.9), \lambda \in \mathfrak{h}_{0}$. Then $\hat{f}_{1}-\hat{g}_{1}$ belongs to $\hat{\mathcal{N}}_{\lambda, A^{+}}$and

$$
0=\tau(\lambda) \Gamma_{0}\left(\hat{f}_{1}-\hat{g}_{1}\right)+\Gamma_{1}\left(\hat{f}_{1}-\hat{g}_{1}\right)=(\tau(\lambda)+M(\lambda)) \Gamma_{0}\left(\hat{f}_{1}-\hat{g}_{1}\right)
$$

implies $\hat{f}_{1}-\hat{g}_{1} \in A_{0} \cap \hat{\mathcal{N}}_{\lambda, A^{+}}$as $\tau(\lambda)+M(\lambda) \neq 0$. Therefore $\hat{f}_{1}=\hat{g}_{1}$.

2. It remains to prove that $\widetilde{A}$ is definitizable over $\Omega^{\prime}$ and that the sign types of $\widetilde{A}$ are $d$-compatible with the sign types of the functions $\tau$ and $M$ in $\Omega^{\prime}$. In this step we show that for every point $\mu \in \Omega^{\prime} \cap \overline{\mathbb{R}}$ there exists an open connected neighbourhood $I_{\mu}$ of $\mu$ in $\Omega^{\prime} \cap \overline{\mathbb{R}}$ such that both components of $I_{\mu} \backslash\{\mu\}$ are of definite type with respect to $\widetilde{A}$.

As the sign types of $\tau$ and $A_{0}$ are $d$-compatible in $\Omega$ Proposition 3.5 implies that the selfadjoint relation $A_{0} \times T_{0}$ is definitizable over $\Omega^{\prime}$. It is straightforward to check that $\left\{\mathbb{C}^{2}, \widehat{\Gamma}_{0}, \widehat{\Gamma}_{1}\right\}$, where

$$
\widehat{\Gamma}_{0}:=\widetilde{\Gamma}_{1}-\Theta \widetilde{\Gamma}_{0}, \quad \widehat{\Gamma}_{1}:=-\widetilde{\Gamma}_{0},
$$


is a boundary value space for $A^{+} \times T^{+}$with $\operatorname{ker} \widehat{\Gamma}_{0}=\widetilde{A}$, corresponding $\gamma$-field

$$
\lambda \mapsto \widehat{\gamma}(\lambda)=\widetilde{\gamma}(\lambda)(\widetilde{M}(\lambda)-\Theta)^{-1}, \quad \lambda \in \mathfrak{h}_{0},
$$

and corresponding Weyl function

$$
\lambda \mapsto \widehat{M}(\lambda)=-(\widetilde{M}(\lambda)-\Theta)^{-1}=-\left(\begin{array}{cc}
M(\lambda) & -1 \\
-1 & -\tau(\lambda)^{-1}
\end{array}\right)^{-1}, \quad \lambda \in \mathfrak{h}_{0} .
$$

In particular

$$
\begin{aligned}
\widehat{M}(\lambda)=\operatorname{Re} \widehat{M}\left(\lambda_{0}\right)+\widehat{\gamma}\left(\lambda_{0}\right)^{+}( & \left(\lambda-\operatorname{Re} \lambda_{0}\right) \\
& \left.+\left(\lambda-\lambda_{0}\right)\left(\lambda-\bar{\lambda}_{0}\right)(\widetilde{A}-\lambda)^{-1}\right) \widehat{\gamma}\left(\lambda_{0}\right)
\end{aligned}
$$

holds for a fixed $\lambda_{0} \in \mathfrak{h}_{0} \cap \Omega^{\prime}$ and all $\lambda \in \mathfrak{h}_{0} \cap \Omega^{\prime}$ (see the proof of Proposition 3.2). By Proposition 3.5 the function $\widehat{M}$ is definitizable in $\Omega$ and the sign types of $\widehat{M}$ and $A_{0} \times T_{0}$ are $d$-compatible in $\Omega^{\prime}$.

Let $\mu \in \Omega^{\prime} \cap \overline{\mathbb{R}}$ and assume e.g. that a onesided open connected neighbourhood $\Delta_{+}$of $\mu$ in $\overline{\mathbb{R}}, \bar{\Delta}_{+} \subset \Omega^{\prime} \cap \overline{\mathbb{R}}$, is of positive type with respect to $A_{0} \times T_{0}$. As the sign types of $\widehat{M}$ and $A_{0} \times T_{0}$ are $d$-compatible in $\Omega^{\prime}$ it is no restriction to assume that $\Delta_{+}$is also of positive type with respect to $\widehat{M}$. Since $A_{0} \times T_{0}$ and $\widetilde{A}$ are both selfadjoint extensions of the symmetric relation $A \times T$ in $\mathcal{K} \times \mathcal{H}$ we have

$$
\operatorname{dim}\left(\operatorname{ran}\left((\widetilde{A}-\lambda)^{-1}-\left(A_{0} \times T_{0}-\lambda\right)^{-1}\right)\right) \leq 2
$$

for all $\lambda \in \mathfrak{h}_{0}$. Let $\Omega_{\Delta_{+}}$be a domain with the same properties as $\Omega, \bar{\Omega}_{\Delta_{+}} \subset$ $\Omega^{\prime}$, such that $\Omega_{\Delta_{+}} \cap \overline{\mathbb{R}}=\Delta_{+}$. It follows from [2, Corollary 2.5] that $\widetilde{A}$ is definitizable over $\Omega_{\Delta_{+}}$and that for every finite union $\delta$ of open connected subsets in $\Delta_{+}, \bar{\delta} \subset \Delta_{+}$, such that the spectral projection $E_{\widetilde{A}}(\delta)$ is defined the space $E_{\widetilde{A}}(\delta)(\mathcal{K} \times \mathcal{H})$ equipped with the inner product $[\cdot, \cdot]_{\mathcal{K} \times \mathcal{H}}$ is a Pontryagin space with finite rank of negativity.

Let $\Omega^{\prime \prime}$ be a domain with the same properties as $\Omega$ such that $\overline{\Omega^{\prime \prime}} \subset \Omega^{\prime}$ and $\bar{\Delta}_{+} \subset \Omega^{\prime \prime} \cap \overline{\mathbb{R}}$. By Theorem 2.6 there exists an $\Omega^{\prime \prime}$-minimal representing relation $S$ for $\widehat{M}$, that is $S$ is a selfadjoint relation in some Krein space $\mathcal{G}$ which is definitizable over $\Omega^{\prime \prime}, \rho(S) \cap \Omega^{\prime \prime}=\mathfrak{h}(\widehat{M}) \cap \Omega^{\prime \prime}$, and with a suitable $\Lambda \in \mathcal{L}\left(\mathbb{C}^{2}, \mathcal{G}\right)$ we have

$$
\widehat{M}(\lambda)=\operatorname{Re} \widehat{M}\left(\lambda_{0}\right)+\Lambda^{+}\left(\left(\lambda-\operatorname{Re} \lambda_{0}\right)+\left(\lambda-\lambda_{0}\right)\left(\lambda-\bar{\lambda}_{0}\right)(S-\lambda)^{-1}\right) \Lambda
$$

for a fixed $\lambda_{0} \in \rho(S) \cap \Omega^{\prime \prime}$ and all $\lambda \in \rho(S) \cap \Omega^{\prime \prime}$. The spectral function of $S$ on $\Omega^{\prime \prime} \cap \overline{\mathbb{R}}$ will be denoted by $E_{S}$ (comp. (2.2)).

In the following we will assume that the point $\lambda_{0}$ in (3.16) and (3.17) belongs to $\rho(\widetilde{A}) \cap \rho(S) \cap \Omega^{\prime \prime}$. This is no restriction. From (3.16) and (3.17) we obtain

$$
\widehat{\gamma}\left(\lambda_{0}\right)^{+} \widehat{\gamma}\left(\lambda_{0}\right)=\Lambda^{+} \Lambda \quad \text { and } \quad \widehat{\gamma}\left(\lambda_{0}\right)^{+}(\widetilde{A}-\lambda)^{-1} \widehat{\gamma}\left(\lambda_{0}\right)=\Lambda^{+}(S-\lambda)^{-1} \Lambda
$$


for all $\lambda \in \rho(\widetilde{A}) \cap \rho(S) \cap \Omega^{\prime \prime}$. Therefore the relation

$$
V:=\left\{\left(\begin{array}{c}
\sum_{k=1}^{n}\left(1+\left(\lambda_{k}-\lambda_{0}\right)\left(S-\lambda_{k}\right)^{-1}\right) \Lambda x_{k} \\
\sum_{k=1}^{n}\left(1+\left(\lambda_{k}-\lambda_{0}\right)\left(\widetilde{A}-\lambda_{k}\right)^{-1}\right) \widehat{\gamma}\left(\lambda_{0}\right) x_{k}
\end{array}\right) \mid \begin{array}{c}
\lambda_{k} \in \rho(S) \cap \rho(\widetilde{A}) \cap \Omega^{\prime \prime} \\
x_{k} \in \mathbb{C}^{2}, k=1, \ldots, n
\end{array}\right\}
$$

is isometric. The assumptions

$$
\mathcal{K}=\operatorname{clsp}\left\{\mathcal{N}_{\lambda, A^{+}} \mid \lambda \in \Omega\right\} \quad \text { and } \quad \mathcal{H}=\operatorname{clsp}\left\{\mathcal{N}_{\lambda, T^{+}} \mid \lambda \in \Omega^{\prime}\right\}
$$

imply

$$
\mathcal{K}=\operatorname{clsp}\left\{\operatorname{ran} \gamma(\lambda) \mid \lambda \in \rho(S) \cap \rho(\widetilde{A}) \cap \Omega^{\prime \prime}\right\}
$$

and

$$
\mathcal{H}=\operatorname{clsp}\left\{\operatorname{ran} \gamma^{\prime}(\lambda) \mid \lambda \in \rho(S) \cap \rho(\widetilde{A}) \cap \Omega^{\prime \prime}\right\},
$$

respectively. From (3.12) and (3.15) we obtain

$$
\mathcal{K} \times \mathcal{H}=\operatorname{clsp}\left\{\widehat{\gamma}(\lambda) x \mid \lambda \in \rho(S) \cap \rho(\widetilde{A}) \cap \Omega^{\prime \prime}, x \in \mathbb{C}^{2}\right\}
$$

and therefore ran $V$ is dense in $\mathcal{K} \times \mathcal{H}$. This implies that $V$ is an isometric operator and the same holds for its closure $\bar{V}$.

Let $\delta$ be a finite union of open connected subsets in $\Delta_{+}, \bar{\delta} \subset \Delta_{+}$, such that the boundary points of $\delta$ in $\overline{\mathbb{R}}$ are of definite type with respect to $\widetilde{A}$. Then $\left(E_{\widetilde{A}}(\delta)(\mathcal{K} \times \mathcal{H}),[\cdot, \cdot]_{\mathcal{K} \times \mathcal{H}}\right)$ is a Pontryagin space with finite rank of negativity. As $\Delta_{+}$is of positive type with respect to $S$ the spectral projection $E_{S}(\delta)$ is defined and $E_{S}(\delta) \mathcal{G}$ equipped with the inner product from $\mathcal{G}$ is a Hilbert space. Writing $E_{S}(\delta)$ and $E_{\widetilde{A}}(\delta)$ as strong limits of the resolvents of $S$ and $\widetilde{A}$, respectively, one verifies that $\bar{V}$ is reduced by $E_{S}(\delta) \mathcal{G} \times E_{\widetilde{A}}(\delta)(\mathcal{K} \times \mathcal{H})$. Then

$$
\bar{V}_{\delta}:=\bar{V} \cap\left(E_{S}(\delta) \mathcal{G} \times E_{\widetilde{A}}(\delta)(\mathcal{K} \times \mathcal{H})\right)
$$

is a closed isometric operator from the Hilbert space $E_{S}(\delta) \mathcal{G}$ with dense range in the Pontryagin space $E_{\widetilde{A}}(\delta)(\mathcal{K} \times \mathcal{H})$. By [12, Theorem 6.2] $\bar{V}_{\delta}$ is bounded and it follows that $E_{\widetilde{A}}(\delta)(\mathcal{K} \times \mathcal{H})$ is a Hilbert space.

Let $\xi \in \delta \cap \sigma(\widetilde{A})$ and choose a sequence $\left(\begin{array}{l}u_{n} \\ v_{n}\end{array}\right) \in \widetilde{A}$ with $\left\|u_{n}\right\|=1$ and $\left\|v_{n}-\xi u_{n}\right\| \rightarrow 0$ for $n \rightarrow \infty$. From

$$
\left(\widetilde{A} \cap\left(\left(I-E_{\widetilde{A}}(\delta)\right)(\mathcal{K} \times \mathcal{H})\right)^{2}-\xi\right)^{-1} \in \mathcal{L}\left(\left(I-E_{\widetilde{A}}(\delta)\right)(\mathcal{K} \times \mathcal{H})\right)
$$

and

$$
\lim _{n \rightarrow \infty}\left\|\left(I-E_{\widetilde{A}}(\delta)\right)\left(v_{n}-\xi u_{n}\right)\right\|=0
$$

we obtain $\left\|\left(I-E_{\widetilde{A}}(\delta)\right) u_{n}\right\| \rightarrow 0$ and $\left\|E_{\widetilde{A}}(\delta) u_{n}\right\| \rightarrow 1$ for $n \rightarrow \infty$. As $E_{\widetilde{A}}(\delta)(\mathcal{K} \times$ $\mathcal{H})$ is a Hilbert space we have

$$
\liminf _{n \rightarrow \infty}\left[u_{n}, u_{n}\right]_{\mathcal{K} \times \mathcal{H}}=\liminf _{n \rightarrow \infty}\left[E_{\widetilde{A}}(\delta) u_{n}, E_{\widetilde{A}}(\delta) u_{n}\right]_{\mathcal{K} \times \mathcal{H}}>0 .
$$


If $\infty$ belongs to $\delta \cap \widetilde{\sigma}(\widetilde{A})$ a similar reasoning shows that $\infty$ is of positive type with respect to $\widetilde{A}$. Therefore $\delta$ is of positive type with respect to $\widetilde{A}$. As this is true for every finite union $\delta$ of open connected subsets in $\Delta_{+}, \bar{\delta} \subset \Delta_{+}$, such that the boundary points of $\delta$ in $\overline{\mathbb{R}}$ are of definite type with respect to $\widetilde{A}$ we conclude that $\Delta_{+}$is also of positive type with respect to $\widetilde{A}$.

Analogously one verifies that a onesided open connected neighbourhood $\Delta_{-}$of $\mu$ in $\overline{\mathbb{R}}, \bar{\Delta}_{-} \subset \Omega^{\prime} \cap \overline{\mathbb{R}}$ which is of negative type with respect to $A_{0} \times T_{0}$ and $\widehat{M}$ is of negative type with respect to $\widetilde{A}$. We have shown that for every point $\mu \in \Omega \cap \overline{\mathbb{R}}$ there is an open connected neighbourhood $I_{\mu}$ in $\overline{\mathbb{R}}$ such that both components of $I_{\mu} \backslash\{\mu\}$ are of the same sign type with respect to $A_{0} \times T_{0}$ and $\widetilde{A}$.

3. It remains to verify that $\widetilde{A}$ belongs to $S^{\infty}\left(\Omega^{\prime} \cap \overline{\mathbb{R}}\right)$. For this we use the relation (3.14). We show first that the selfadjoint relation $T_{1}=\operatorname{ker} \Gamma_{1}^{\prime}$ in $\mathcal{H}$ is definitizable over $\Omega^{\prime}$. As the function

$$
\begin{aligned}
-\tau(\lambda)^{-1}=\operatorname{Re}\left(-\tau\left(\lambda_{0}\right)^{-1}\right)+ & \tau\left(\bar{\lambda}_{0}\right)^{-1} \gamma^{\prime}\left(\lambda_{0}\right)^{+}\left(\left(\lambda-\operatorname{Re} \lambda_{0}\right)\right. \\
& \left.+\left(\lambda-\lambda_{0}\right)\left(\lambda-\bar{\lambda}_{0}\right)\left(T_{1}-\lambda\right)^{-1}\right) \gamma^{\prime}\left(\lambda_{0}\right) \tau\left(\lambda_{0}\right)^{-1}
\end{aligned}
$$

(see (3.11) and the proof of Proposition 3.2) is definitizable in $\Omega$ and the selfadjoint relation $T_{0}$ is definitizable over $\Omega^{\prime}$ the same considerations as in step 2 of the proof applied to $-\tau^{-1}, T_{0}$ and $T_{1}$ instead of $\widehat{M}, A_{0} \times T_{0}$ and $\widetilde{A}$ show that every point $\mu \in \Omega^{\prime} \cap \overline{\mathbb{R}}$ has an open connected neighbourhood $I_{\mu}$ in $\overline{\mathbb{R}}$ such that both components of $I_{\mu} \backslash\{\mu\}$ are of definite type with respect to $T_{1}$. By (3.5) we have

$$
\left(T_{1}-\lambda\right)^{-1}=\left(T_{0}-\lambda\right)^{-1}+\gamma^{\prime}(\lambda)\left(-\tau(\lambda)^{-1}\right) \gamma^{\prime}(\bar{\lambda})^{+}
$$

for all $\lambda \in \mathfrak{h}(\tau) \cap \mathfrak{h}\left(\tau^{-1}\right)$. Since $-\tau^{-1}$ is definitizable in $\Omega$ the nonreal spectrum of $T_{1}$ in $\Omega^{\prime}$ does not accumulate to points in $\Omega^{\prime} \cap \overline{\mathbb{R}}$. The growth properties of $-\tau^{-1}$ (see Definition 2.5) and the resolvent of $T_{0}$ imply $T_{1} \in S^{\infty}\left(\Omega^{\prime} \cap \overline{\mathbb{R}}\right.$ ) and therefore $T_{1}$ is definitizable over $\Omega^{\prime}$.

As the sign types of $-\tau^{-1}$ and $A_{0}$ are $d$-compatible in $\Omega$ (see the proof of Proposition 3.5 (iii)) it follows that $A_{0} \times T_{1}$ is definitizable over $\Omega^{\prime}$. The relation

$$
(\widetilde{A}-\lambda)^{-1}=\left(A_{0} \times T_{1}-\lambda\right)^{-1}+\widetilde{\gamma}(\lambda) \widehat{M}(\lambda) \widetilde{\gamma}(\bar{\lambda})^{+}, \quad \lambda \in \mathfrak{h}_{0},
$$

(cf. (3.14)) and the growth properties of $\widehat{M}$ and the resolvent of $A_{0} \times T_{0}$ show $\widetilde{A} \in S^{\infty}\left(\Omega^{\prime} \cap \overline{\mathbb{R}}\right)$. This completes the proof of Theorem 3.6.

Remark 3.7. Let $\Omega, \Omega^{\prime}, A \subset A_{0}, \tau$ and $T_{0}$ be as in Theorem 3.6 and let $\Delta$ be an open connected subset in $\overline{\mathbb{R}}, \bar{\Delta} \subset \Omega^{\prime} \cap \overline{\mathbb{R}}$, which is of positive (negative) 
type with respect to $A_{0}$ and $\tau$. As $T_{0}$ is an $\Omega^{\prime}$-minimal representing relation for $\tau$ it follows that $\Delta$ is of positive (negative) type with respect to the selfadjoint relation $A_{0} \times T_{0}$ in $\mathcal{K} \times \mathcal{H}$. From [2, Corollary 2.5] we obtain that for every finite union $\delta$ of open connected subsets in $\overline{\mathbb{R}}, \bar{\delta} \subset \Delta$, such that the spectral projection $E_{\widetilde{A}}(\delta)$ corresponding to $\widetilde{A}$ in $(3.8)$ and the set $\delta$ is defined, $\left(E_{\widetilde{A}}(\delta)(\mathcal{K} \times \mathcal{H}),[\cdot, \cdot]_{\mathcal{K} \times \mathcal{H}}\right)$ is a Pontryagin space with finite rank of negativity (positivity). This can also be deduced from [3, Theorem 4.1].

Remark 3.8. The assumption $\mathcal{H}=\operatorname{clsp}\left\{\mathcal{N}_{\lambda, T^{+}} \mid \lambda \in \Omega^{\prime}\right\}$ in Theorem $3.6 \mathrm{im}-$ plies that the selfadjoint extension $\widetilde{A}$ in (3.8) satisfies the minimality condition

$$
\mathcal{K} \times \mathcal{H}=\operatorname{clsp}\left\{\left(1+\left(\lambda-\lambda_{0}\right)(\widetilde{A}-\lambda)^{-1}\right)\{k, 0\} \mid k \in \mathcal{K}, \lambda \in \rho(\widetilde{A}) \cap \Omega^{\prime}\right\}
$$

for some fixed $\lambda_{0} \in \rho(\widetilde{A}) \cap \Omega^{\prime}$. This can be verified as in [3, Proof of Theorem 4.1]. Let $A \in \widetilde{\mathcal{C}}(\mathcal{K})$ be as in Theorem 3.6 and assume that $\widetilde{B}$ is a selfadjoint extension of $A$ in some Krein space $\mathcal{K} \times \mathcal{H}^{\prime}$ which is definitizable over $\Omega^{\prime}$ such that the compressed resolvent of $\widetilde{B}$ onto $\mathcal{K}$ yields a solution of (3.9). Then $\left.P_{\mathcal{K}}(\widetilde{B}-\lambda)^{-1}\right|_{\mathcal{K}}$ and $\left.P_{\mathcal{K}}(\widetilde{A}-\lambda)^{-1}\right|_{\mathcal{K}}$ coincide. If $\widetilde{B}$ fulfils the minimality condition (3.18) with $\mathcal{K} \times \mathcal{H}$ and $\rho(\widetilde{A}) \cap \Omega^{\prime}$ replaced by $\mathcal{K} \times \mathcal{H}^{\prime}$ and $\rho(\widetilde{B}) \cap \Omega^{\prime}$, respectively, and we choose $\lambda_{0} \in \rho(\widetilde{A}) \cap \rho(\widetilde{B}) \cap \Omega^{\prime}$, then

$$
W:=\left\{\left(\begin{array}{l}
\sum_{i=1}^{n}\left(1+\left(\lambda_{i}-\lambda_{0}\right)\left(\widetilde{A}-\lambda_{i}\right)^{-1}\right)\left\{k_{i}, 0\right\} \\
\sum_{i=1}^{n}\left(1+\left(\lambda_{i}-\lambda_{0}\right)\left(\widetilde{B}-\lambda_{i}\right)^{-1}\right)\left\{k_{i}, 0\right\}
\end{array}\right) \mid \begin{array}{c}
\lambda_{i} \in \rho(\widetilde{A}) \cap \rho(\widetilde{B}) \cap \Omega^{\prime}, \\
k_{i} \in \mathcal{K}, i=1,2, \ldots, n
\end{array}\right\}
$$

is a densely defined isometric operator in $\mathcal{K} \times \mathcal{H}$ with dense range in $\mathcal{K} \times \mathcal{H}^{\prime}$ and the same holds for its closure $\bar{W}$. We denote the local spectral functions of $\widetilde{A}$ and $\widetilde{B}$ by $E_{\widetilde{A}}$ and $E_{\widetilde{B}}$, respectively. Let $\Delta \subset \Omega^{\prime} \cap \overline{\mathbb{R}}$ be a closed connected set such that $E_{\widetilde{A}}(\Delta)$ is defined. Then also $E_{\widetilde{B}}(\Delta)$ is defined and $\bar{W}$ is reduced by

$$
E_{\widetilde{A}}(\Delta)(\mathcal{K} \times \mathcal{H}) \times E_{\widetilde{B}}(\Delta)\left(\mathcal{K} \times \mathcal{H}^{\prime}\right) .
$$

The closed isometric operator

$$
\bar{W}_{\Delta}:=\bar{W} \cap\left(E_{\widetilde{A}}(\Delta)(\mathcal{K} \times \mathcal{H}) \times E_{\widetilde{B}}(\Delta)\left(\mathcal{K} \times \mathcal{H}^{\prime}\right)\right)
$$

intertwines the resolvents of

$$
\widetilde{A}_{1}:=\widetilde{A} \cap\left(E_{\widetilde{A}}(\Delta)(\mathcal{K} \times \mathcal{H})\right)^{2} \quad \text { and } \quad \widetilde{B}_{1}:=\widetilde{B} \cap\left(E_{\widetilde{B}}(\Delta)\left(\mathcal{K} \times \mathcal{H}^{\prime}\right)\right)^{2},
$$

i.e. for $\lambda \in \rho\left(\widetilde{A}_{1}\right) \cap \rho\left(\widetilde{B}_{1}\right) \cap \Omega^{\prime}$ and $x \in \operatorname{dom} \bar{W}_{\Delta}$ we have

$$
\bar{W}_{\Delta}\left(\widetilde{A}_{1}-\lambda\right)^{-1} x=\left(\widetilde{B}_{1}-\lambda\right)^{-1} \bar{W}_{\Delta} x .
$$

In particular, the ranks of positivity and negativity of the inner products on the subspaces $E_{\widetilde{A}}(\Delta)(\mathcal{K} \times \mathcal{H})$ and $E_{\widetilde{B}}(\Delta)\left(\mathcal{K} \times \mathcal{H}^{\prime}\right)$ coincide. 
If, in addition to the assumptions above, $\left(E_{\widetilde{A}}(\Delta)(\mathcal{K} \times \mathcal{H}),[\cdot, \cdot]_{\mathcal{K} \times \mathcal{H}}\right)$ is a Pontryagin space, then $E_{\widetilde{B}}(\Delta)\left(\mathcal{K} \times \mathcal{H}^{\prime}\right)$ equipped with the inner product from $\mathcal{K} \times \mathcal{H}^{\prime}$ is also a Pontryagin space and by $[12$, Theorem 6.2$]$ the operator $\bar{W}_{\Delta}$ is an isometric isomorphism of $E_{\widetilde{A}}(\Delta)(\mathcal{K} \times \mathcal{H})$ onto $E_{\widetilde{B}}(\Delta)\left(\mathcal{K} \times \mathcal{H}^{\prime}\right)$, i.e. $\widetilde{A}_{1}$ and $\widetilde{B}_{1}$ are isometrically equivalent.

The case that the function $\tau$ is a real constant is excluded in Theorem 3.6. In this case we have the following theorem.

Theorem 3.9. Let $\Omega$ be a domain as in the beginning of Section 3.2 and let $A$ be a closed symmetric relation with defect one in the Krein space $\mathcal{K}$ such that $\mathcal{K}=\operatorname{clsp}\left\{\mathcal{N}_{\lambda, A^{+}} \mid \lambda \in \Omega\right\}$ holds. Assume that there exists a selfadjoint extension $A_{0}$ of $A$ which is definitizable over $\Omega$. Let $\left\{\mathbb{C}, \Gamma_{0}, \Gamma_{1}\right\}$ be a boundary value space for $A^{+}, A_{0}=\operatorname{ker} \Gamma_{0}$, denote by $\gamma$ and $M$ the corresponding $\gamma$-field and Weyl function, respectively, and let $\tau$ be a real constant.

Then the relation $A_{-\tau}=\operatorname{ker}\left(\Gamma_{1}+\tau \Gamma_{0}\right)$ is a selfadjoint extension of $A$ in $\mathcal{K}$ which is definitizable over $\Omega$. The sign types of $M$ and $A_{-\tau}$ are $d$-compatible in $\Omega$. For every $k \in \mathcal{K}$ and every $\lambda \in \Omega \cap \mathfrak{h}(M) \cap \mathfrak{h}\left((M+\tau)^{-1}\right)$ a solution of the boundary value problem

$$
f_{1}^{\prime}-\lambda f_{1}=k, \quad \tau \Gamma_{0} \hat{f}_{1}+\Gamma_{1} \hat{f}_{1}=0, \quad \hat{f}_{1}=\left(\begin{array}{c}
f_{1} \\
f_{1}^{\prime}
\end{array}\right) \in A^{+},
$$

is given by

$$
\begin{aligned}
& f_{1}=\left(A_{-\tau}-\lambda\right)^{-1} k=\left(A_{0}-\lambda\right)^{-1} k-\gamma(\lambda)(M(\lambda)+\tau)^{-1} \gamma(\bar{\lambda})^{+} k, \\
& f_{1}^{\prime}=\lambda f_{1}+k .
\end{aligned}
$$

Proof. The proof of Theorem 3.9 is a modification of the proof of Theorem 3.6. Note first that the relation (3.4) and $\mathcal{K}=\operatorname{clsp}\left\{\operatorname{ran} \gamma(\lambda) \mid \lambda \in \Omega \cap \rho\left(A_{0}\right)\right\}$ imply that the Weyl function $M$ is not identically equal to a constant. Here it is obvious that the resolvent of $A_{-\tau}$ yields a solution of the boundary value problem (3.19) (compare (3.1), (3.2) and (3.5)). As in step 2 and step 3 of the proof of Theorem 3.6 the function $\lambda \mapsto-(M(\lambda)+\tau)^{-1}$, which by $[1$, Theorem 2.3] is definitizable in $\Omega$, can be regarded as the Weyl function corresponding to a boundary value space $\left\{\mathbb{C}, \hat{\Gamma}_{0}, \hat{\Gamma}_{1}\right\}$ for $A^{+}$with $A_{-\tau}=\operatorname{ker} \hat{\Gamma}_{0}$. Now the same arguments as in the proof of Theorem 3.6 show that $A_{-\tau}$ is definitizable over $\Omega$. The details are left to the reader.

\section{REFERENCES}

[1] T.Ya. Azizov, P. Jonas: On Locally Definitizable Matrix Functions, submitted.

[2] J. Behrndt, P. Jonas: On Compact Perturbations of Locally Definitizable Selfadjoint Relations in Krein Spaces, to appear in Integral Equations Operator Theory. 
[3] J. Behrndt, P. Jonas: Boundary Value Problems with Local Generalized Nevanlinna Functions in the Boundary Condition, submitted.

[4] V.A. Derkach: On Weyl Function and Generalized Resolvents of a Hermitian Operator in a Krein Space, Integral Equations Operator Theory 23 (1995), 387-415.

[5] V.A. Derkach: On Generalized Resolvents of Hermitian Relations in Krein Spaces, J. Math. Sci. (New York) 97 (1999), 4420-4460.

[6] V.A. Derkach, S. Hassi, M.M. Malamud, H.S.V. de Snoo: Generalized Resolvents of Symmetric Operators and Admissibility, Methods Funct. Anal. Topology 6 (2000), 2453.

[7] V.A. Derkach, M.M. Malamud: Generalized Resolvents and the Boundary Value Problems for Hermitian Operators with Gaps, J. Funct. Anal. 95 (1991), 1-95.

[8] V.A. Derkach, M.M. Malamud: The Extension Theory of Hermitian Operators and the Moment Problem, J. Math. Sci. (New York) 73 (1995), 141-242.

[9] A. Dijksma, H.S.V. de Snoo: Symmetric and Selfadjoint Relations in Krein Spaces I, Operator Theory: Advances and Applications 24 (1987), Birkhäuser Verlag Basel, 145-166.

[10] A. Dijksma, H.S.V. de Snoo: Symmetric and Selfadjoint Relations in Krein Spaces II, Ann. Acad. Sci. Fenn. Math. 12, 1987, 199-216.

[11] V.I. Gorbachuk, M.L. Gorbachuk: Boundary Value Problems for Operator Differential Equations, Kluwer Academic Publishers, Dordrecht (1991).

[12] I.S. Iohvidov, M.G. Krein, H. Langer: Introduction to the Spectral Theory of Operators in Spaces with an Indefinite Metric, Mathematical Research 9 (1982), Akademie-Verlag Berlin.

[13] P. Jonas: On a Class of Unitary Operators in Krein Space, Operator Theory: Advances and Applications 17 (1986), Birkhäuser Verlag Basel, 151-172.

[14] P. Jonas: A Class of Operator-Valued Meromorphic Functions on the Unit Disc, Ann. Acad. Sci. Fenn. Math. 17 (1992), 257-284.

[15] P. Jonas: Operator Representations of Definitizable Functions, Ann. Acad. Sci. Fenn. Math. 25 (2000), 41-72.

[16] P. Jonas: On Locally Definite Operators in Krein Spaces, in: Spectral Theory and Applications, Theta Foundation, (2003).

[17] P. Jonas: On Operator Representations of Locally Definitizable Functions, submitted.

[18] H. Langer: Spectral Functions of Definitizable Operators in Krein Spaces, Functional Analysis Proceedings of a Conference held at Dubrovnik, Yugoslavia, November 2-14, 1981, Lecture Notes in Mathematics, 948, Springer Verlag Berlin-Heidelberg-New York, 1982, 1-46. 